\title{
Estructura de la industria maquiladora de exportación: un ensayo de interpretación y búsqueda de conceptos
}

\author{
Bernardo González Aréchiga \\ El Colegio de la Frontera Norte
}

\section{Resumen}

El propósito de este trabajo es plantear una visión crítica de las aproximaciones disponibles provistas por los estudiosos del tema y propone un método de análisis basado en la posibilidad de utilizar información sobre insumos, servicios, capital, activos financieros y ventas para estudiar el sector. Sugiere una estructura económica desagregada que permita incorporar detalles importantes relacionados con el intercambio económico. Realiza una redefinición en la que las restricciones para tipificar a una empresa como maquiladora son mayores, y más aún para considerar a ésta una empresa internacional. Es deseable calcular los conceptos de producto internacional y producto interno de todas las actividades productivas; aunque el problema es, en gran medida de costo de oportunidad.

Palabras clave: industria maquiladora de exportación, insumos, intercambio económico, producto nacional, divisas.

\begin{abstract}
This job has the objective to establish a critical vision of the available approaches provided by the experts on the topic, proposing a rigorous analysis that is based on the possibility to use information regarding production goods, services, capital, financial assets, and sales in order to perform a research on this sector. It suggests a disaggregated economical structure that allows the inclusion of important details related to the economical exchange. It performs a redefinition that includes the bigger restrictions required to typify a corporation as a maquila industry, and even more if this company was to be considered an international corporation. It is desirable to calculate the concepts on international product as well as the internal product of each one of the productive activities. However, this is mostly a problem of high cost of opportunities.
\end{abstract}

Keywords: exporting maquila industry, production goods, economical exchange, domestic product, national currency. 


\title{
ESTRUCTURA DE LA INDUSTRIA MAQUILADORA DE EXPORTACION: UN ENSAYO DE INTERPRETACION Y BUSQUEDA DE CONCEPTOS
}

\author{
Realizado por: \\ Bernardo González Arćchiga*
}

\section{INTRODUCCION}

El presente trabajo es un ensayo exploratorio sobre la estructura económica de la industria maquiladora de exportación localizada en México. Su propósito es explorar conceptos, variables e instrumentos de medición que permitan llegar a una interpretación coherente de algunos de los elementos claves de esta industria. El ensayo también pretende hacer una crítica constructiva de la información que se recopila y publica acerca de la industria y de los marcos teóricos y conceptuales que tradicionalmente se han utilizado al analizar la industria.

El puntode partida oel motivo del presente trabajoes el diagnóstico de las limitaciones y problemas que tienen la mayoría de los estudios sobre las maquiladoras. Los más importantes son los siguientes:

1. Adoptar un marco teórico de referencia paradigmático que es excesivamente general y presenta problemas para contrastarse directamente con la evidencia empírica.

2. Frecuentemente, los estudios parten de la conecpeión de que la actividad maquiladora es un reflejo de procesos tecnológicos y comerciales que suceden en los países desarrolladose involucran únicamente en forma instrumental a los menos desarrollados. Sin embargo, no se estudia al primer mundo sino al tercero, provocando una ruptura entre la naturaleza de los fenomenos estudiados y la de los datos utilizados. Existc, por lo tanto, una falta de correspondencia entre las hipótesis y la información que se discute.

3. Existe una inadecuada explicación de las razones quesustentan la distribución mundial de la actividad maquiladora; el énfasis se pone normalmente en el crecimiento de esta actividad en cada país sin una referencia clara de los factores que determinan su distribución.

- Director đel Departamento de Estuđios Económicos đel COLEF 
4. Ignoran el análisis de los procesos y los medios de competencia, no sólo entre países aspirantes a ser maquiladores, como sc mencionó anteriormente, sino también entre empresas que maquilan en distintos países con regímenes comerciales diferentes (y, en consecuencia, elasticidades distintas) yentre empresas que participan en procesos de maquila y las que no lo hacen.

Eston sugiere los siguientes planteamientos: a) ¿Cómo se determina el precio por los servicios de maquila?, b) ¿Cuál es la magnitud de las cuasi-rentas que genera la actividad maquiladora y cuál es su papel en la estrategia comercial global?

5. La mayoría de los estudios sufren por la escasez de datos porque: a) la información oficial sobre el sector es demasiado agregada; b) las encucstas no se basan en muestras verdaderamente representativas; c) es sumamente difícil relacionar información sobre la empresa matriz y la filial, de modo de establecer una relación rigurosa sustentada en la teoría de los dos tipos de empresa; d) es difícil aislar las empresas que participan en la subcontratación (ya sea como matrices o filiales) del resto de las unidades productivas de cada país, y e) es difícil relacionar, por medio de procesos de mercado, las empresas que participan en la subcontratación con el resto de las unidades de cada país.

El trabajo se divide en dos partes. En la primera se presenta una reflexión general acerca de la definición de la industria maquiladora, contando con cl apoyo de un sencillo modelo matemático. En esa sección se pretende sentar las bases para la discusión más profunda de conceptos que aparecen posteriormente. En la segunda parte se elabora un modelo más complejo que se utiliza para analizar los conceptos de maquiladoras, subcontratación y la transferencia internacional de procesos productivos. También se discuten críticamente algunas de las principales limitaciones de la información sobre el sector, recopilada tanto por Estados Unidos como por México, y se analizan sus diferencias. El trabajo se termina con una discusión de la contribución de las maquiladoras al producto nacional y al producto interno de México; el argumento consiste en que la evaluación del papel de la industria al desarrollo de las regiones fronterizas del norte y del país, requiere de un entendimiento más cabal de las relaciones económicas de la actividad maquiladora. 


\section{PRIMERA PARTE}

\section{Reflexiones acerca de la definición de la industria maquiladora.}

Si bien es cierto que existe consenso acerca de los criterios posibles para la clasificación de las industrias maquiladoras, no lo hay en lo que se refiere a la definición general del término. La industria es normalmente identificada en las investigaciones por medio de su carácter legal, más que por sus características generales. Un estudio que se diferencia de la mayoría de los casos es el de Jorge Carrillo y Alberto Hernández que definen la maquiladora de acuerdo a cuatro características que mezclan aspectos legales (como su filiación por medio de lazos de propiedad), la intensidad de las relaciones de mercados externas, y la intensidad de los horarios de trabajo. En esta definición, como en la mayoría de las que se presentan en la literatura, subyace el aspecto tecnológico-comercial de la reubicación geográfica de procesos productivos y la revisión de estrategias de división internacional del trabajo. Este aspecto es tan importante que, en muchos casos, se pretende que la simple evocación del proceso global sea suficiente para definir el fenómeno maquilador.

En la mayoría de los estudios se define la industria maquiladora en esos términos que son: o bien, demasiado estrechos para captar la naturaleza del fenómeno (como es el caso de la definición legal basada en el registro federal correspondiente), o bien, demasiado gencrales, ya que se define por medio del proceso global que representan (como es el caso que lo identifica mecánicamente con el proceso de "internacionalización" del capital y la "nueva división internacional del trabajo"). Ambas vías de definición dejan la explicación del fenómeno maquilador al contexto de un proceso más general, que en pocas ocasiones es rigurosamente definido.

La definición legal de las maquiladoras tienc la ventaja y la limitación de estar sujeta al cambio. Cuando se adopta este criterio, se tiene implícitamente la concepción de que la legislación abre cl espacio económico en el que operan las compañías mexicanas y extranjerasen el territoriomexicano. Lalegislación se ve, por lo tanto, como la estructura que condiciona la forma de operación de la industria ya que ésta adecúa sus estrategias comerciales, laborales, productivas y de operación en la medida en que la legislación lo permite. Por lo tanto, la definición legal depende de decretos y estatutos, pero más críticamente, de la interpretación del espacio 
económico y estratégico que abre a las entidades productivas que constantemente buscan mayor flexibilidad para sus operaciones. Lo que se estudia normalmente es la legislación, pero lo que se debe estudiar en realidad, de acuerdo a este enfoque, son las posibilidades estratégicas que se abren para "los usuarios de la legislación".

La definicion de maquiladora en este enfoque, necesariamente cambia al cambiar la legislacion, por lo que pueden ser inestables los estudios que se basan en esos criterios. Además estos estudios no se pueden considerar completos si no explican los cambios ocurridos en los periodos estudiados, o no se demuestra que ellos alteran radicalmente las definiciones operativas implícitas.

El segundo enfoque, normalmente se basa en la descripción de las estrategias adoptadas por las compañías internacionales que utilizan los servicios de plantas maquiladoras. Dentro de este conjunto sobresalen varias definiciones basadas en propiedad, organización, control o en las características de los flujos de mercado. Un ejemplo particularmente ciudadosoes de la Encuesta Nacional a Plantas Maquiladoras del Centro de Investigación y Docencia Económica (CIDE), que se reporta en el trabajo de Jesús Tamayo y José Luis Fernández. En ese trabajo se adoptó como criterio de definición el origen de los insumos productivos ya que "una empresa sería considerada como maquiladora, cuando el valor de los insumos recibidos de otra empresa para la que realizara operaciones productivas representase, al menos, $10 \%$ del valor total de los insumos utilizados en la producción total...". En forma semejante se define el origen de la maquiladora: "Una empresa maquiladora sería considerada internacional, siempre que el valor de los insumos importados representara como mínimo $51 \%$ del valor total de los insumos utilizados en la realización de dicha operación..." (Tamayo y Fernández, 1983).

En forma semejante, la participación del capital extranjero en la maquiladora es loque define si es filial o contratada.Así, "Una empresa sería considerada como filial, siempre que el capital social de la misma fuese propiedad en $25 \%$ o más de la empresa que le hubiere encargado la realización dela operación productiva" (Tamayo y Fernández,1983). Si no se cumple esta condición la empresa se clasifica como contratada.

Estas definiciones son suceptibles a un tratamiento ciudadoso de conceptos y dan origen a una clasificación rigurosa de los distintos tipos de empresas. Para desarrollar este punto conviene definir las siguientes variables: 


\section{Indices :}

i representa la empresa con la que la unidad productiva bajo estudio tiene relaciones comerciales de transformación o subcontratación.

n representa empresas con las que la unidad productiva bajo estudio tiene relaciones comerciales que no se pueden clasificar como de transformación o subcontratación.

j representa la nacionalidad de la empresa contratante i o $\mathrm{n}$, si $j=1$ entonces indica que $i$ o n son empresas nacionales, si $j=\mathbf{2}$ son extranjeras (este índice se puede subdividir para tomar en consideración el país extranjero en concreto).

\section{Variables}

I $(\mathrm{i}, \mathrm{j})$ mide la cantidad de insumos productivos que utiliza la empresa en cuestión procedentes de una industria tipo i, con la que existen relaciones de subcontratación,

$T(i, j)$ servicios de transformaciónque ofrece la industriaestudiada a empresas tipo $\mathrm{i}$,

K (i,j) bienes de capital (bienes físicos productivos) que utiliza la empresa estudiada procedentes de la empresa tipo i,

C (i,j) valor de los activos financieros transferidos por la empresa tipo $\mathrm{i}$ localizada en $\mathrm{j}$ a la empresa estudiada,

V representa el valor de las ventas efectuadas por la empresa en cuestión sin tomar en cuenta actividades de transformación.

La misma definición se puede aplicar con los cambios en el tipo de empresa de origen para las variables $I(n, j), T(n, j)$ y $C(n, j)$.

Con las variables anteriores se pueden definir una serie de cocientes que permiten clasificar las empresas de acuerdo a su vinculación, por vía de compra de insumos y bienes de capital, con otras empresas nacionales y extranjeras. Con estas variables se puede definir no sólo a las empresas maquiladoras, sino también toda una clase de unidades productivas distintas. La investigación económica de este tipo de entidades productivas se puede centrar en el estudio de la composición industrial resultante de diversos esquemas de clasificación, la estabilidad de estos esquemas y los cambios que redundan en un reacomodo de las empresas en las clases estudiadas. No se puede olvidar que el principal objetivo de cualquier clasificación es contribuir a entender el comportamiento del universo por medio de la comprensión de los elementos, claramente identificables, que lo conforman. Conviene recordar aquí que la calidad de cualquier esquema de clasificación 
depende de la claridad de las fronteras que definen los componentes individuales, su estabilidad y las diferencias en el comportamiento o en las propiedades de esos componentes.

La figura 1 representa los flujos descritos por las definiciones aritméticas anteriores, su propósito es presentar en forma sencilla las interrelaciones entre cada uno de los flujos y presentar una imagen de conjunto. Esta función es importante ya que las relaciones entre las partes es lo que con frecuencia se utiliza como criterios de definición de la industria maquiladora y de clasificación de sus componentes. De hecho, las definiciones utilizadas por Tamayo y Fernández se pueden presentar de mancra formal por medio de las siguientes relaciones matemáticas:

1. maquiladoras

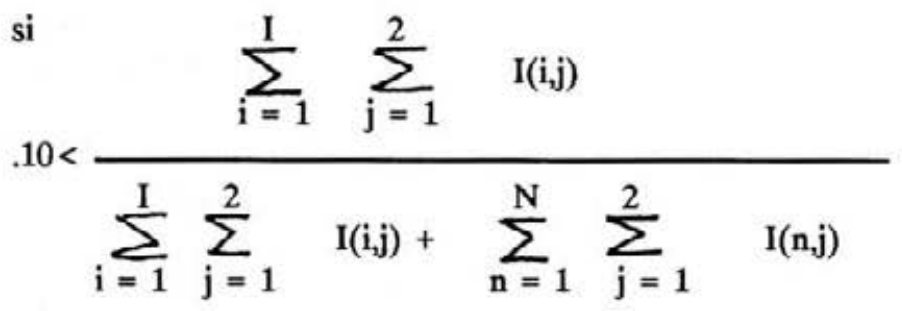

2. maquiladora internacional

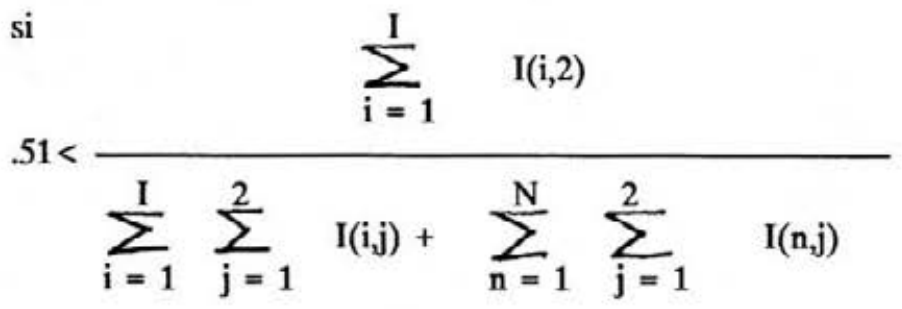




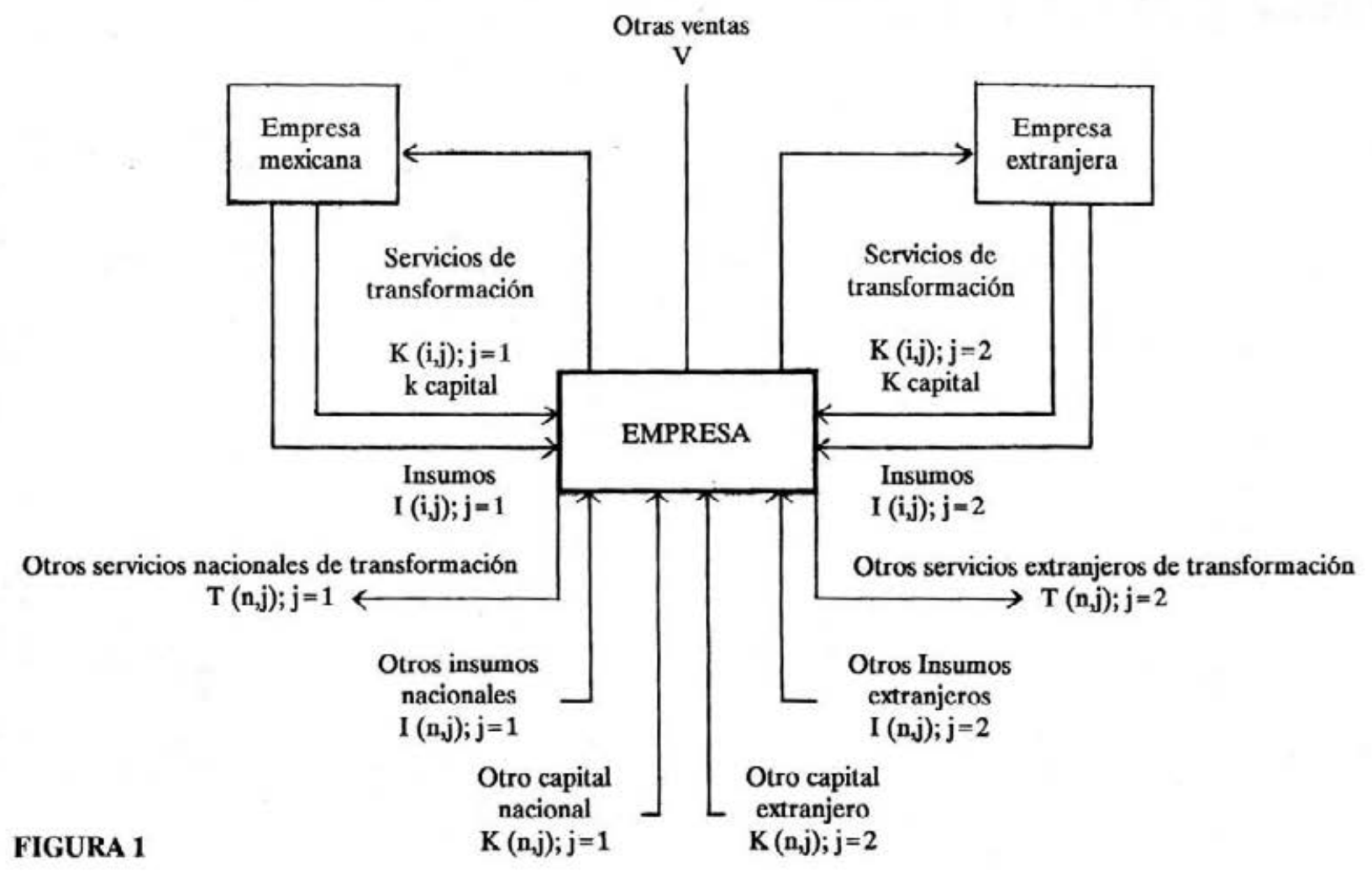


3. maquiladora internacional filial

$$
0.25<\frac{K\left(i^{*}, 2\right)}{\sum_{i=1}^{I} \sum_{j=1}^{2} K(i, j)+\sum_{n=1}^{N} \sum_{j=1}^{2} K(n, j)}
$$

4. maquiladora internacional contratada

$$
0.25>\frac{K\left(i^{*}, 2\right)}{\sum_{i=1}^{I} \sum_{j=1}^{2} K(i, j)+\sum_{n=1}^{N} \sum_{j=1}^{2} K(n, j)}
$$

La variable $\mathrm{i}^{*}$ satisface la siguiente condición:

$$
\mathrm{T}\left(\mathrm{i}^{*}, 2\right)>\mathrm{T}(\mathrm{i}, 2) \text { para toda } \mathrm{i}=1, \ldots \mathrm{I} .
$$

Cabe notar que la definición 1 de la empresa maquiladora no es necesariamente consistente con una definición que se basara en el porcentaje del producto total ( o del valor agregado) que se destina a operaciones de transformación. Esta definición alternativa se puede escribir de la siguiente manera:

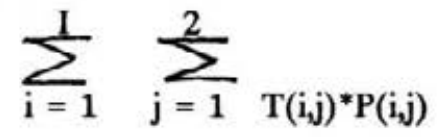
$>.10$

$$
\sum_{i=1}^{I} \sum_{j=1}^{2} T(i, j) * P(i, j)+\sum_{n=1}^{N} \sum_{j=1}^{2} T(n, j) * P(n, j)+V^{*} P V
$$

Esta definición alternativa y la descrita por la ecuación 1 serían perfectamente equivalentes si: 1) la medición de producto total y de 
valor agregado en ambas actividades se realizara con los mismos criterios, y 2) las funciones de maquila y de transformación fuesen igualmente productivas. Si en ambas condiciones se cumplieran, el criterio basado en insumo y el basado en producto final llevarían a clasificaciones equivalentes. Si éste no fuera el caso, como cabría csperar para el caso de la maquiladora en México, entonces sería necesario explicar en mayor detalle por qué se debe preferir el primer criterio sobre el segundo y explicar en qué condiciones se debería seleccionar cl segundo. Esta es una labor que no realizaron los autores mencionados.

La discusión de las definiciones anteriores no se debe entender como una defensa de los conceptos, sino como un ejemplo del uso de un método riguroso de clasificación y como cvidencia de que no existe una clasificación de las empresas bajo cstudio que pueda tomarse acríticamente como suficiente. Más bien, lo que se sugiere anteriormente es que es necesario retroceder hasta el campo más básico de las definiciones. Si bien es cierto que no es necesario iniciar una profunda labor de exégesis acerca de la cmpresa maquiladora, función que sin duda se debe realizar en investigaciones más ambiciosas, sí es necesario cuestionar las definiciones más comunes e iniciar la búsqueda de conceptos más estructurales.

Las definiciones de Tamayo y Fernández se pueden resumir en forma esquemática en la figura 2. El propósito de la figura es mostrar que las fronteras entre los cuatro tipos de empresas maquiladoras no son estables, sino que pueden ser cruzadas aún sin existir cambios estructurales en las empresas. Es decir, la clasificación no cumple el objeto de captar elementos estructurales del funcionamiento de los mercados de bienes intermedios y de capital. Variaciones en el tipo de cambio (la devaluación) puede alterar el valor contable de activos e insumos nacionales y externos moviendo, de hecho, a la empresa estudiada de un recuadro de la figura 2 a otro, sin que ese cambio hubiera resultado de un verdadero cambio estructural. La inflación nacional puede tener el mismoefecto en la clasificación ya que modifica los precios relativos y cambia el significado de las fronteras o los límites de la clasificación de empresas.

Los comentarios anteriores no deben interpretarse como una crítica a los autores citados; por el contrario, el esfuerzo de proponer una clasificación precisa pone en evidencia los problemas que aquejan a la mayoría de los estudiosen el área. Quizás los estudios más afectados por las críticas anteriores son aquéllos que adoptan las definiciones más 


\begin{tabular}{|c|c|c|c|c|c|}
\hline & & \multicolumn{2}{|c|}{ Maquiladora } & \multicolumn{2}{|c|}{ No Maquiladora } \\
\hline & & Nacional & Extranjera & Nacional & Extranjcra \\
\hline & Filial & $\begin{array}{l}\% \\
\text { de } \\
\mathrm{c} \\
\mathrm{a} \\
\mathrm{p} \\
\mathrm{i} \\
\mathrm{t} \\
\mathrm{a} \\
\mathrm{l}\end{array}$ & & 4 & \\
\hline & Contratada & $\begin{array}{l}e \\
x \\
t \\
r \\
a \\
n \\
j \\
e \\
r \\
o\end{array}$ & $\begin{array}{l}\text { \% de insumos de } \\
\text { origen externo }\end{array}$ & & \\
\hline 12 & & & & & \\
\hline
\end{tabular}


ambiguas, ya que éstos no contribuyen a la comprensión de las maquiladoras por ignorar una de las principales formas de reducir la varianza en el comportamiento económico (clasificandoy estudiando en forma separada los elementos que componen el universo) y por adoptar definiciones que no esclarecen conceptualmente la naturaleza de los fenómenos estudiados.

Las reflexiones anteriores pretenden generar una polémica interna y sugerir preguntas para proseguir la investigación. Como ejemplo de las preguntas que surgen se pueden citar las siguientes:

1. ¿Cómo han cambiado los criterios legales que definen el campo de acción de la industria maquiladora?

2. iSe puede considerar que las definiciones legales y las definiciones funcionales son consistentes entre sí?

3. ¿Si hay que optar por un solo criterio de clasificación, cuál debe de seleccionarse?

4. ¿En qué estudios conviene adoptar cada uno de estos criterios?

5: ¿Es factible, como se sugiere en el texto anterior, utilizar los estatutos legales para definir el campo económico de acción abierto a las maquiladoras?

6. ¿Son los cambios legales suficientemente importantes como para permitir un estudio del cambio en cl ámbito de acción de las maquiladoras a través del tiempo?

7. ¿Cómo interactúan las distintas leyes nacionales para formar un espectro completo de posibilidades económicas de la industria?

8. ¿Es conveniente utilizar el marco legal para comparar industrias de transformación y maquiladoras, y para entender los posibles medios y motivos de transición de una forma legal a otra para empresas concretas?

9. ¿Qué tan robustas son las leyes nacionales de maquiladoras para tolerar cambios no estructurales en las condiciones económicas como son los precios o el tipo de cambio?

10. De existir un reacomodo no deseable en la clasificación de empresas maquiladoras, iquiénes ganan y quiénes pierden con estos cambios? icuáles son los costos para el Estado mexicano y cómo se distribuyen? iafectan la base gravable y los mecanismos de control?

A estas preguntas se pueden agregar muchas otras, las que se listan aquí solamente indican las posibles direcciones que puede tomar un investigación en las líneas propuestas. 


\section{SEGUNDA PARTE}

Estructura y medición de las relaciones económicas de la industria maquiladora.

Después de haber discutido los aspectos generales de la definición de la maquiladora y haber planteado algunas preguntas acerca del significado de los conceptos y criterios de clasificación, procedercmos en esta segunda parte a sugerir una estructura económica más detallada. El propósito es presentar una estructura desagregada en que se puedan incorporar algunos de los detalles más importantes de los intercambios económicos en que participa la maquiladora. La descripción misma de las relaciones pone en evidencia lo poco que conocemos del funcionamientode la industria, sugiere distintos medios para recopilar información nueva, plantear preguntas y diseñar esquemas de investigación.

La actividad maquiladora se caracteriza por la composición multinacional de los insumos del proceso productivo y por el tratamiento arancelario especial en cada uno de los países que intervienen en la producción. La complejidad del intercambio económico que ocurre dentro de la estructura de los contratos de maquila, están en gran medida ocultos detrás de la información excesivamente general que se reporta en las estadísticasoficiales de laindustria. Eldinamismo interno se pierde en las grandes tendencias de crecimiento a largo plazo y en las variaciones cíclicas, en el número de plantas y el personal ocupado. En estos grandes números se pierden: los cambios en la composición de los insumos; el aprovechamiento de las leyes especiales de los países involucrados; los cambios en la productividad; las variaciones en los procesos tecnológicos y en la composición de los productos; los cambios en la participación del capital autóctono; los cambios en la vulnerabilidad de la industria y la dependencia.

Por esa razón, esta sección se dedica al análisis abstracto de las principales definiciones que se utilizan para describir el fenómeno maquilador en México. El propósito central de esta discusión es sugerir indicadores más precisos y conceptos más adecuados para el análisis de las llamadas maquiladoras.

\section{Nomenclatura y definición de términos:}

La definición de los conceptos básicos de subcontratación, maqui- 
ladoras, transferencia internacional de procesos, valor agregado y valor total, requiere de la utilización de siete variables, tres formas de medición y algunos parámetros adicionales de intercambio. Esta información aparece tabulada en el cuadro 1 . Las principales variables son:

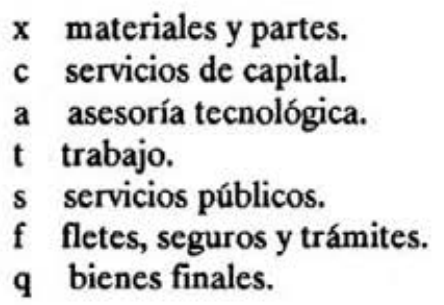

Cada una de cllas se puede representar por sus unidades físicas utilizando la letra minúscula; por su precio unitario de mercado $o$, en su defecto, por su precio de transferencia utilizandola letra P seguida por la letra minúscula que designa la variable, y por su valor monetario total representado por la letra mayúscula. Todos los precios y valores menctarios totales están evaluados en dólares.

La definición completa de los insumos requiere de seis subíndices que indican el tipo de insumo para cada categoría (índice i), el bien final a que se incorpora (índice m), el lugar de origen (índice j), la firma de origen (índice k), el tratamiento arancelario que recibe en México el insumo (índice n), y el trato arancelario que recibe en Estados Unidos el producto (índice l). En su conjunto estos f́ndices describen la naturaleza del insumo original y del bien a que se incorpora; toda esa información es necesaria para poder definir conceptos más precisos para medir el desempeño de la llamada industria maquiladora. El significado de cada subíndice se presenta en el cuadro 2.

Puede notarse en el cuadro 1 que las variables: trabajo, servicios públicos y fletes, seguros y trámites no tienen los índices k, n yl, ya que dada la estructura legal vigente de las maquiladoras, no importa ni la firma de origen ni el tratamiento arancelario en México y Estados Unidos.

El producto final, en forma semejante a los insumos, se define por medio de 4 índices que indican: el tipo de bien (índice $\mathrm{m}$ ), el lugar de destino (índicej), la firma de destino (índice k). yel trato arancelario del producto en Estados Unidos (índice e). Su significado es el mismo 
CUADRO 1. Definición de las variables centrales del análisis y los índices que determinan sus propiedades.

\begin{tabular}{|c|c|c|c|c|c|c|c|c|c|}
\hline & \multicolumn{4}{|c|}{ S f m bolos } & \multicolumn{5}{|c|}{ Indices } \\
\hline & $\begin{array}{l}\text { Cantidades } \\
\text { físicas }\end{array}$ & $\left|\begin{array}{c}\text { Valor } \\
\text { unitario }\end{array}\right|$ & $\begin{array}{l}\text { Valor } \\
\text { total }\end{array}$ & \begin{tabular}{|l} 
Tipo de \\
insumos
\end{tabular} & $\begin{array}{l}\text { Bien } \\
\text { final } \\
\end{array}$ & $\begin{array}{c}\text { Lugar de } \\
\text { origen }\end{array}$ & $\begin{array}{c}\text { Firma de } \\
\text { origen }\end{array}$ & $\begin{array}{l}\text { Trato arancelario } \\
\text { de Mexico }\end{array}$ & $\begin{array}{c}\text { Trato arancelario } \\
\text { de E.U. }\end{array}$ \\
\hline \multicolumn{10}{|l|}{ I n s u mos } \\
\hline Materiales y partes & $\mathrm{x}$ & Px & $\mathrm{x}$ & i & $\mathrm{m}$ & j & k & $\mathbf{r}$ & 1 \\
\hline Capital & c & $\mathrm{Pc}$ & C & i & $\mathrm{m}$ & j & k & n & 1 \\
\hline Asesoría tecnológica & a & $\mathrm{Pa}$ & A & i & $\mathrm{m}$ & j & k & n & 1 \\
\hline Trabajo & t & Pt & $\mathbf{T}$ & i & $\mathrm{m}$ & j & - & - & - \\
\hline Servicios páblicos & $\mathrm{s}$ & Ps & s & $\mathrm{i}$ & $\mathrm{m}$ & j & - & - & - \\
\hline Fletes, seguros y trámites & $\mathrm{f}$ & Pf & $\mathrm{F}$ & $\mathrm{i}$ & $\mathrm{m}$ & j & - & - & $\cdots$ \\
\hline \multicolumn{10}{|l|}{ Productos } \\
\hline Bienes finales & q & $\mathrm{Pq}$ & Q & & $\mathrm{m}$ & j & k & - & 1 \\
\hline \multicolumn{10}{|l|}{ Otras variables } \\
\hline $\begin{array}{l}\text { Tasa de ganancia } \\
\text { contractual (porcentual). }\end{array}$ & 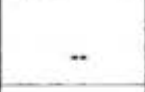 & $\mathrm{g}$ & - & - & m & j & k & - & 1 \\
\hline Tasa de mark up administrativa & - & r & -. & - & m & $-j$ & - & - & $\cdots$ \\
\hline Utilidad & - & - & $\mathrm{U}$ & .. & $\mathrm{m}$ & j & k & - & -1 \\
\hline Tarifa (porcentual) & .. & t & -. & - & m & - & - & - & - \\
\hline
\end{tabular}


que se asigna a los índices de los insumos y se detalla también en el cuadro 2.

Antes de proceder a sugerir algunas definiciones de conceptos más elaborados como el de valor agregado, conviene aclarar algunos aspectos de los índices de las variables de insumos y productos. Los que se refieren al tipo de insumo (i), bien final (m) y firma de origen (k), son índices que simplemente listan progresivamente, sin que importe el orden, los tipos de insumos y firmas respectivamente. Loque se pretende con ellos es asociar los insumos con los productos en que se incorporan y distinguir los compradores; no se busca, en primera instancia, describir la naturaleza de los bienes ni las características de los compradores. En contraste, en el caso de los índices del lugar de origen o destino (índice j) y el trato arancelario en México y Estados Unidos (índices n y l), se busca captar elementos concretos de la subcontratación; estos índices no representan una secuencia arbitraria de bienes y servicios distintos, sino que cada número de la secuencia tiene un significado concreto. En el caso del índice $j$, por ejemplo, el número uno significa que el origen del insumo es el interior de México; el dos, que es la frontera de México; el tres que es Estados Unidos y el cuatro, otro país.

Además de las variables anteriores se requieren cuatro más para complementar las definiciones. Estas son: la tasa de ganancia contractual de la planta subcontratada (g); la tasa mark up administrativa que se carga en aduana para compensar costos indirectos(r); la utilidad total derivada de la venta del bien $m$ en los distintos mercados (U), y la tarifa que carga Estados Unidos a la importación de los bienes procesados ( $\mathrm{t}$ ). Estas variables son indispensables para medir tanto el valor agregado y $\mathrm{cl}$ total como $\mathrm{cl}$ monto del gravamen exportado.

\section{Subcontratación}

Se dice que en una empresa participa en labores de subcontratación si hay una firma $k^{*}$ que abastece un insumo i para la producción de $\mathrm{m}^{*}$ tal que :

(1) $\mathrm{X}\left(\mathrm{i}^{*}, \mathrm{~m}^{*}, \mathrm{j}, \mathrm{k}^{*}, \mathrm{n}, \mathrm{l}\right) \cdot \mathrm{Q}\left(\mathrm{m}^{*}, \mathrm{j}, \mathrm{k}^{*}, \mathrm{l}\right)>0$

para algún valor de $\mathrm{j}, \mathrm{n}$ y l; es decir, independientemente del tipo de insumo, su procedencia y destino y su tratamiento arancelario.

Una definición alternativa es la siguiente: existe subcontratación 
CUADRO 2. Códigos

\begin{tabular}{|c|c|c|c|c|c|c|}
\hline Valor & $\begin{array}{c}\text { i } \\
\text { Tipo de } \\
\text { insumo }\end{array}$ & $\stackrel{\mathrm{m}}{\text { Bien final }}$ & \begin{tabular}{l}
\multicolumn{1}{c}{$\mathrm{j}$} \\
Lugar de \\
origen o \\
destino
\end{tabular} & \begin{tabular}{l}
\multicolumn{1}{c}{$\mathbf{k}$} \\
Firma de \\
origen o \\
destino
\end{tabular} & \begin{tabular}{l}
\multicolumn{1}{c}{$\mathrm{n}$} \\
Trato aran- \\
celario a \\
importación \\
de insumos \\
México
\end{tabular} & \begin{tabular}{l}
\multicolumn{1}{c}{1} \\
Trato aran- \\
celario a \\
exportación \\
de insumos \\
E.U.
\end{tabular} \\
\hline 1 & $\begin{array}{c}\text { Insumo } \\
1\end{array}$ & $\begin{array}{c}\text { Bien } \\
1\end{array}$ & $\begin{array}{l}\text { México } \\
\text { interior }\end{array}$ & Firma 1 & $\begin{array}{l}\text { No exento } \\
\text { de impuesto }\end{array}$ & $\begin{array}{l}\text { No exento } \\
806 / 807\end{array}$ \\
\hline 2 & $\begin{array}{c}\text { Insumo } \\
2\end{array}$ & $\begin{array}{c}\text { Bien } \\
2\end{array}$ & $\begin{array}{l}\text { México } \\
\text { frontera }\end{array}$ & Firma 2 & $\begin{array}{l}\text { Exento de } \\
\text { impuesto }\end{array}$ & $\begin{array}{l}\text { Exento dc } \\
\text { impuesto } \\
806 / 807\end{array}$ \\
\hline 3 & $\begin{array}{c}\text { Insumo } \\
3\end{array}$ & $\begin{array}{c}\text { Bien } \\
3\end{array}$ & $\begin{array}{l}\text { Estados } \\
\text { Unidos }\end{array}$ & Firma 3 & - & Exento GSP \\
\hline 4 & $\begin{array}{c}\text { Insumo } \\
4\end{array}$ & $\begin{array}{c}\text { Bien } \\
4\end{array}$ & $\begin{array}{l}\text { Otro } \\
\text { país }\end{array}$ & Firma 4 & $\overline{--}$ & $\overline{--}$ \\
\hline$\overline{5}$ & $\begin{array}{c}\text { Insumo } \\
5\end{array}$ & $\begin{array}{c}\text { Bien } \\
5\end{array}$ & $\overline{--}$ & Firma 5 & - & - \\
\hline 6 & $\underset{6}{\text { Insumo }}$ & $\begin{array}{c}\text { Bicn } \\
6\end{array}$ & - & Firma 6 & -- & - \\
\hline
\end{tabular}


si la firma estudiada tiene relaciones comerciales con una firma $\mathrm{k}^{*}$ en un producto $\mathrm{m}^{*}$, tal que:

(2.a) $\mathrm{C}\left(\mathrm{i}, \mathrm{m}^{*}, \mathrm{j}, \mathrm{k}^{*}, \mathrm{n}, \mathrm{l}\right) \cdot \mathrm{Q}\left(\mathrm{m}^{*}, \mathrm{j}, \mathrm{k}^{*}, \mathrm{l}\right)>0$

o

(2.b) a(i,m*j,k,n,l, $), Q\left(m^{*}, j, k, l\right)>0$

Para algún valor de i, j,n, y l.

La subcontratación es internacional, de acuerdo a los criterios anteriores, si las condiciones (1) o (2) se cumplen para valores de $j \geqslant 3$.

La primera definición de subcontratación asume que la relación más importante es la que se establece por medio de la obtención de materiales y parte para su elaboración posterior o ensamble. La scgunda, supone que cl vínculo que detcrmina si existe o no un convenio de subcontratación, es la prestación de servicios de capital por parte de la empresa que recibe el producto "maquilado" a aquélla que lo produce. Finalmente, la tercera definición liga la prestación de servicios de asesoría tecnológica del cliente al provecdor. En todos los casos anteriores se afirma que la transferencia de un producto de una empresa a otra, constituye una instancia de subcontratación si se acompaña de un flujo de insumos o servicios productivos que va en dirección opuesta al producto.

De acuerdo a las definiciones anteriores, solo puede existir la subcontratación si la transacción del bien o servicio final es antecedida por una relación entre las empresas contratantes a nivel del proceso productivo. Es decir, se da una doble relación en la que ambas empresas son demandantesa la vez; por lotanto, el contrato de subcontratación, tal y como se define, implica dos transacciones y no es simplemente la venta de un bien o un servicio.

Esta relación comercial dual puede tomar muchas formas. Un caso extremo es el que resulta si ambas transacciones se llevan a cabo en forma independiente y cada una se negocia de acuerdo a un convenio tradicional de compra-venta. Otra posibilidad, que es la que da lugar a los convenios de maquila, es la contratación simultánea de las dos transacciones.

En concreto, la subcontratación constituye un contrato de maquila (Martínez Tarragó, et al) si: 
(3.a) el producto maquilado $\mathrm{m}^{*}$ utiliza los materiales y partes $\mathrm{i}^{*}$ provistos por la empresa $\left(\mathrm{k}^{*}, \mathrm{j}^{*}\right)$ sin que su valor sea un elemento que afecte al proceso de decisión de la empresa productora,

(3.b) el precio del bien maquilado se define en el contrato de tal forma que compensa los costos de producción y además proporciona una tasa de ganancia $\mathrm{g}\left(\mathrm{m}^{*}, \mathrm{j}^{*}, \mathrm{k}^{*}, \mathrm{l}\right)$ sobre los costos totales; es decir:

$$
\begin{aligned}
& P q\left(m^{*}, j^{*}, k^{*}, l\right) \cdot q\left(m^{*}, j^{*}, k^{*}, l\right)= \\
& \begin{aligned}
Q\left(m^{*}, j^{*}, k^{*}, l\right)=\left(l+g\left(m^{*}, j^{*}, k^{*} l\right)\right)^{*} \\
\sum_{\substack{k=1 \\
k=k^{*}}}^{K} \sum_{j=1}^{L} \sum_{j=1}^{J} \sum_{n=1}^{N} \sum_{i=1}^{J} X\left(i, m^{*}, j^{*}, k^{*}, n, l\right)+C\left(i, m^{*}, j^{*}, k^{*}, n, l\right) \\
\\
+A\left(i, m^{*}, j, k, n, l\right)+T\left(i, m^{*}, j^{*}\right) \\
+S\left(i, m^{*}, j^{*}\right)+F\left(i, m^{*}, j^{*}\right)
\end{aligned}
\end{aligned}
$$

El inciso (a) indica que la firma $\left(\mathrm{j}^{*}, \mathrm{k}^{*}\right)$, provee el insumo $\mathrm{i}^{*}$ para la producción de $\mathrm{m}^{*}$ sin transferir la propiedad del insumo a la maquiladora. Igualmente, la empresa maquiladora no paga por los servicios de capital, ni la asesoría tecnologica y administrativa que recibe de la empresa $\left(\mathrm{j}^{*}, \mathrm{k}^{*}\right)$; el precio de esos insumos de la producción es irrelevante en la toma de decisiones.

El inciso (b) muestra que la ganancia de la maquiladora depende de una tasa implícita de utilidad (variable $\left.\mathrm{g}\left(\mathrm{m}^{*} \mathrm{j}^{*}, \mathrm{k}^{*}, \mathrm{l}\right)\right)$ sobre los costos normales de manufactura, ensamble o transformacion del producto $\mathrm{m}^{*}$. Se excluye de la estimación total de costos el de los insumos, asesorías, servicios de capital y otros servicios aportados por la firma $\left(\mathrm{j}^{*}, \mathrm{k}^{*}\right)$ que recibe el bien final. La utilidad, en otras palabras, no depende directamente del precio de mercado del bien $\mathrm{m}^{*} \mathrm{o}$ de los insumos y servicios, sino de su valor contable de transferencia que se calcula con base en el costo unitario normal de producción, -en el caso de empresas con la relación matriz filial-, o de la negociación de los servicios productivos, a destajo-en el caso de empresas contratadas. En otras palabras, el precio por unidad maquilada realmente determina, dada la eficiencia productiva de la planta, el costo de los demás insumos, la ganancia como porcentaje del costo directo total de producción que es la cantidad que aparece en la sumatoria. 
De hecho, uno de los retos teóricos más importantes a que se enfrenta el estudio de las llamadas maquiladoras es comprender el proceso de determinación del valor de los servicios productivos; la respuesta de la industria a los cambios en las condiciones de mercado depende de la naturaleza del proceso de negociación. En particular, la capacidad generadora de divisas de la actividad no sólo depende de la evolución de los mercados de los bienes finales parcialmente producidos en el país, sino también del tipo de contrato.

\section{Maquiladoras.}

La importancia relativa de los contratos de maquila para una empresa que participa en la subcontratación determina que una empresa, como un todo, se puede o no llamar maquiladora. Martínez Tarrag6 y sus coautores proponen que se clasifique una empresa como maquiladora si los contratos de maquila son de tal magnitud que absorben cuando menos el $10 \%$ de los insumos totales. Es decir:

$$
\sum_{m=m^{*}}^{M} \sum_{j=j^{*}}^{J} \sum_{k=k^{*}}^{K} \sum_{i=1}^{I} \sum_{n=1}^{N} \sum_{l=1}^{L} x\left(i, m^{*}, j^{*}, k^{*}, n, l\right)
$$

(4) IAM = $>.10$

$$
\sum_{i=1}^{I} \sum_{m=1}^{M} \sum_{j=1}^{J} \sum_{k=1}^{K} \sum_{n=1}^{U} \sum_{I=1}^{L} x(i, m, j, k, n, l)
$$

Se puede definir el mismo concepto tomando el valor total del producto $\mathrm{m}^{*}$, o sea la variable $\mathrm{Q}\left(\mathrm{m}^{*}, \mathrm{j}, \mathrm{k}, 1\right)$, como referencia para medir la relevancia de los contratos de maquila; también sería posible utilizar el valor agregado, la cantidad total de servicios de capital o cualquier otra variable. Conviene preguntarse también por qué la tasa de 10 por ciento es el criterio de selección. Podría pensarse que cualquier contrato de maquila convierte a la empresa en maquiladora, o que solamente aquellas en que dominan este tipo de contratos merecen el nombre de maquiladoras. Este punto es crucial para poder entender la participación de empresas que estaban originalmente fuera de los procesos de subcontratación, pero que los han adoptado como una 
estrategia de mercadotecnia para enfrentar la caída en la demanda interna.

Aquí se propone que se considere como maquiladora cualquier empresa que incurre en las prácticas de subcontratación. La relación (4) no debe usarse como un criterio de clasificación, sino como la definición de un índice de participación en la actividad maquiladora al que podríamos llamar IAM. De hecho, los datos de las maquiladoras deberían distinguir entre las empresas que tienen unvalor de IAM igual a uno (es decir, empresas $100 \%$ maquiladoras) y el resto; para el segundo grupo de empresas sería conveniente contar con la tasa media de utilización de capacidad instalada. Estos dos indicadores serían muy útiles para entender el proceso de crecimiento de la actividad maquiladora, y para entender la subcontratación como respuesta a una situación de crisis de demanda y/o como respuesta a condiciones nuevas de relación con el exterior.

Lo que conviene enfatizar es que si bien la actividad de maquila se puede dar en todos los niveles de la producción y entre todo tipo de empresas, el concepto de maquiladora se reserva normalmente a las que involucran contratos de carácter internacional. De hecho, las estadísticas del ramo se refieren única y exclusivamente a este último tipo de contratos.

\section{Maquiladora internacional:}

La maquiladora internacional es aquella empresa que tiene contratos de maquila con una empresa $k^{*}$ localizada en el extranjero $\left(j^{*}=3,4\right)$. Es decir, es una empresa que cumple las condiciones (1), (3.a) y (3.b) con la contraparte $\left(\mathrm{k}^{*}, \mathrm{j}^{*}\right)$ localizada fucra del país.

En el trabajo de Trinidad Martínez Tarragó et. al. se reserva el término de maquilador internacional a aquellas firmas que reciben del extranjero más de la mitad de los insumostotales; éstas se subdividen en filiales y contratadas dependiendo del origen del capital. Una maquiladora se considera filial si más de la cuarta parte del capital social es propiedad de la empresa $\left(\mathrm{j}^{*}, \mathrm{k}^{*}\right)$ que hubiese encargado la realización de la operación productiva; las demás se clasifican como contratadas. En este trabajo se utiliza una definición más general de la maquiladora internacional, aunque se retoma la composición del capital al discutir los conceptos de producto nacional $\mathrm{c}$ interno de las actividades de producción internacional conjunta.

La definición anterior implica que no todos los programas de 
importación temporal para la exportación que entran en la clasificación mexicana de maquila representan, según las definiciones anteriores, una forma de subcontratación o, menos aún, de maquila. Solamente caen en la primera clasificación aquellos programas en que la firma abastecedora de insumos es la que compra los bienes procesados, y en la segunda si no hay una transferencia real de la propiedad de los insumos.

\section{Transferencia internacional de procesos productivos:}

La importancia de las definiciones es evidente cuandotratamos de pasar del concepto de maquiladora internacional al de producción internacional conjunta y cuando utilizamos las estadísticas de comercio exterior de México y Estados Unidos como indicadores de la magnitud y la evolución del fenomeno.

Una primera aproximación consiste en estimar que cualquier eventode importación temporal para la transformación yexportación que involucre a una sola firma externa $\left(\mathrm{j}^{*}, \mathrm{k}^{*}\right)$, como proveedora de materias primas y compradora del producto final,constituye una instancia de producción internacional conjunta. Esta interpretación asignaría un papel secundario a los procesos tecnológicos y comerciales, a los que se atribuye el surgimiento de los acuerdos de subcontratación. Por otro lado, si asigna el papel motriz a la tecnología y a las estrategias comerciales, es necesario separar las maquiladoras internacionales de acuerdo a los procesos productivos que realizan en México como parte de los procesos globales de producción. Esta clasificación permitiría identificar aquellos procesos que merecen ser considerados como una verdadera transferencia internacional.

Aún si se opta por la definición anterior, debe enfatizarse que se toma como referencia la definición general de subcontratación y no la de maquila. La diferencia es importante ya que todo contrato de maquila es un acto de subcontratación, pero no a la inversa. $\mathrm{La}$ diferencia reside en la forma de propiedad sobre los insumos y en el mecanismo para determinar los precios de los bienes transformados. En el caso de la maquila no existe una venta de productos e insumos propiamente dicha, sino una prestación de un servicio productivo; la base de valoración es distinta en ambas circunstancias y con ésta el funcionamiento del sistema de precios.

La relevancia de tomar en cuenta la tecnología y las estrategias comerciales para acotar los casos de subcontratación que obedecen 
a una producción internacional conjunta, es que se pueden observar los mismos síntomas generales si la transferencia busca la reducción de costos de producción (como es el caso de la mayor parte de las empresas mexicanas subcontratantes con empresas de Estados Unidos) o si busca las apertura de mercados nacionales o extranjeros (como es el caso de empresas mexicanas subcontratantes con empresas japonesas). Sin embargo, el comportamiento y la evolución de estos dos tipos de operación pueden ser radicalmente distintos; además, la lógica de atacar mercados nuevos es más semejante a la de establecer firmas transnacionales en mercados protegidos que la de alcanzar una nueva división internacional del trabajo. Se cometería un error de agregación si se atribuye todo el fenómeno de subcontratación o de maquila a cualquiera de los dos procesos de ajuste internacional.

Las definiciones anteriores permiten identificar 8 tipos de asociaciones entre empresas ligadas por medio de relaciones insumo producto, según se resumen en la figura 3 . Los principales criterios de clasificación son cuatro: la subcontratación, la maquila, la producción internacional conjunta para reducir costos y para penetrar mercados. Pudiéramos agregar los conceptos de maquiladora internacional, contratada y filial; que dividirían en tres los conjuntos 3 y 6 de la figura. Con esta división adicional tendríamos 12 tipos de relaciones bilaterales entre empresas productivas que complementan sus procesos productivos, 10 de ellas de carácter internacional. Cada tipo de relación es, en principio, distinta en su respuesta a condiciones coyunturales y estructurales.

\section{Estadísticas y definición de maquiladoras en México:}

El uso de estadísticas oficiales de México y Estados Unidos para estudiar la evolución de la producción internacional conjunta, requicre del entendimiento de las definiciones implícitas de los conceptos. Las estadísticas que México reporta no se refieren directamente al fenómeno de la subcontratación, la subcontratación internacional o a los contratos de maquila, sino a la actividad productiva que se registra bajo el "régimen maquilador" que establece el Decreto de Maquiladoras. La selección y el acopio de información se hace sobre la base del registro de empresas y los programas maquiladores que resumen información sobre los contratos y los programas independientes de producción de las empresas. 


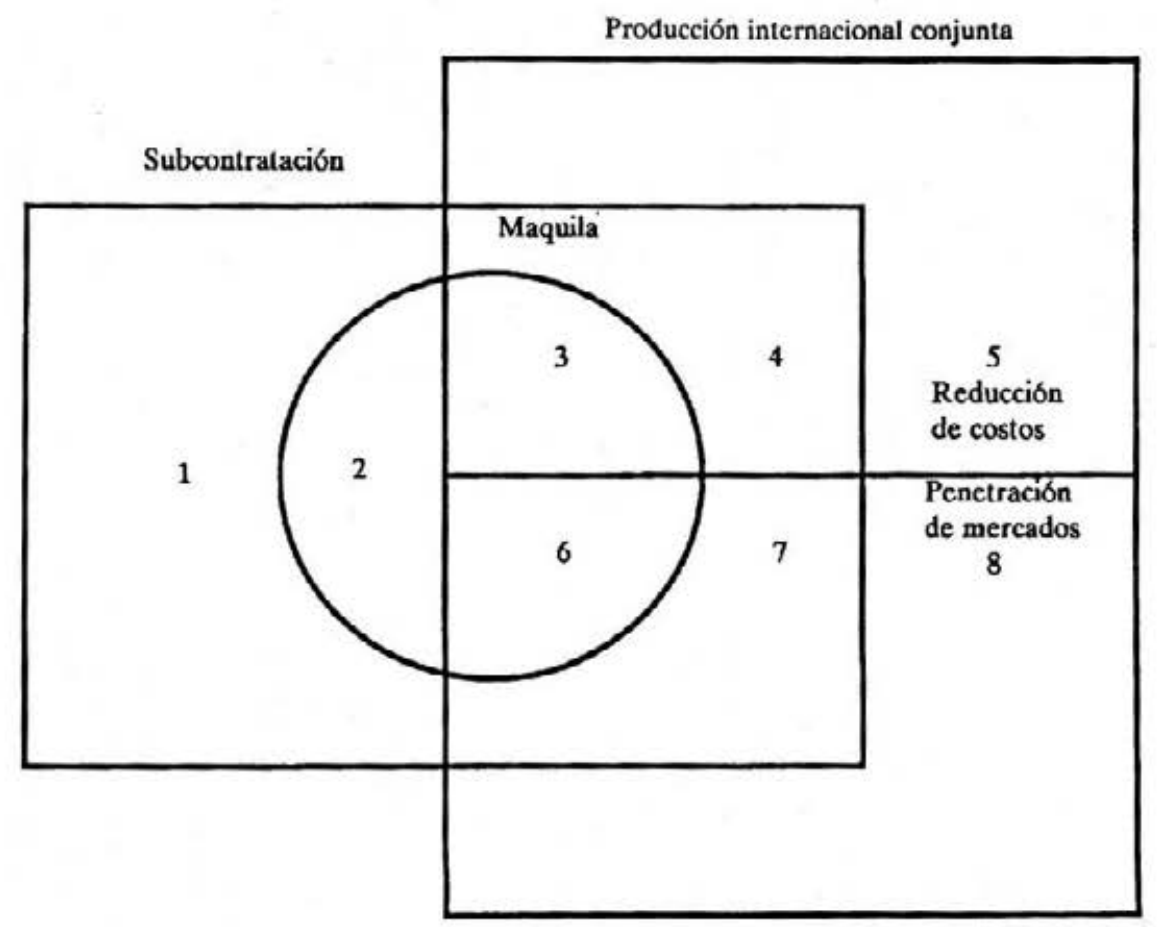

FIGURA 3 
Las estadísticas mexicanas reportan 34 variables, 9 de ellas derivadas de las 25 variables primarias de acuerdo a los criterios de agregación. Las variables cubren el número de establecimientos, el personal ocupado de acuerdo a sus características, las remuneraciones totales y su composiciòn, el origen y la naturaleza de materiales y partes, y la composición del valor agregado y los gastos diversos. Todos estos datos se presentan solamente en términos de valor monetarios y en agregados por localidad o por rama industrial. Solamente en el caso de la información sobre el empleo se presentan datos sobre las unidades físicas, en número de trabajadores y horas laboradas totales además de las compensaciones. Esta falta de desglose del valor total entre el valor unitario y las cantidades físicas, es una de las limitaciones más graves de la información. Otra limitación consiste en que la organización de los datos permite analizar solamente la localización geográfica o la rama industrial, pero no es posible analizar ambos factores simultáneamente.

Sin embargo, estas objeciones a la estructura de los datos es menos importante que la que se refiere a su naturaleza. De acuerdo a nuestras definiciones anteriores, la información sobre el sector maquilador combina, en proporciones desconocidas y probablemente cambiantes, programas de subcontratación internacional, programas de maquilay programas de importación temporal para la exportación que no caen en las definiciones anteriores. Las ventajas que ofrece el régimen maquilador (en cuanto a trámites aduaneros, créditos y administración) pucden garantizar que no se cometa un error grave de subestimación por omisión; pero la flexibilidad que ha imprimido la ley al concepto lleva a incluir actividades que no se pueden considerar propiamente maquiladoras.

La definición por registro se puede expresar formalmente de la siguiente forma: una empresa se incluye en las estadísticas de maquiladoras si tiene cuando menos un bien $\mathrm{m}^{*}$ que utiliza un insumo $\mathrm{i}^{*}$ de procedencia extranjeras $(j \geqq 3)$ que reciba un tratamiento de libre importación y exportación en México $(n=1)$; independientemente de la identidad de la firma de origen del insumo importado, de la firma de destino del producto y del trato arancelario que se le asigne al producto en el exterior. Es decir, existe un producto $\mathrm{m}^{*}$ que utiliza un insumo importado $\mathrm{i}^{*}$ tal que: 
para algún valor de $\mathrm{k} \mathrm{y} \mathrm{l,} \mathrm{y} \mathrm{para} \mathrm{j} \geqq 3$.

Conviene señalar que esta definición:

(a) No requiere que la firma $\mathrm{k}$ que produce el insumo sea la misma que compra el producto final, por lo tanto la condición (5) no se refiere necesariamente a un contrato de subcontratación que aparece en la relación (1).

(b) No estipula si se transfiere la propiedad del insumo $\mathrm{i}^{*}$ a la empresa que produce $\mathrm{m}^{*}$, ni cómo se asigna un precio al producto final; por lo tanto, el contrato puede o no ser verdaderamente de maquila según la definición (3).

(c) Sin embargo, sí exige que la relación comercial se establezca con una firma productiva externa.

Las estadísticas mexicanas sobre maquiladoras se refieren a la producción y los insumos asociados con la transformación, ensamble o produccion del bien (o los bienes) $\mathrm{m}^{*}$ de cada empresa que cumplen con la condición (5). Los demás programas productivos de la empresa nose registran en estas estadísticas, aun cuando constituyen el contexto productivo en que se da el fenómeno estudiado.

Uno de los datos centrales de las estadísticas bajo el rubro maquilador, es el valor agregado en la producción del bien $\mathrm{m}^{*}$ registrado en el programa maquilador $(n=2)$, que se estima agregando el valor de los materiales y partes mexicanas, los servicios de capital, la asesoría tecnológica y administrativa y el trabajo, servicios públicos, fletes, seguros y trámites y lasutilidades a la empresa en México. Es decir:

$$
\begin{aligned}
& \text { (6) VA }\left(m^{*}\right)=\sum_{i=1}^{I} \sum_{j=1}^{J} \sum_{k=1}^{K} \sum_{n=1}^{N} \sum_{l=1}^{L} X\left(i, m^{*}, j, k, n, l,\right)+ \\
& C\left(i, m^{*}, j, k, n, l,\right)+A\left(i, m^{*}, j, k, n, l, l\right)+ \\
& T\left(i, m^{*}, j, l,\right)+S\left(i, m^{*}, j, l\right)+F\left(i, m^{*}, j, l\right)+V\left(m^{*}, j, k, l\right) \\
& -\sum_{i=1}^{I} \sum_{j=3}^{4} \sum_{k=1}^{K} \sum_{l=1}^{L} \quad X\left(i, m^{*}, j, k, 2,1\right)+C\left(i, m^{*}, j, k, 2,1\right)+ \\
& A\left(i, m^{*}, j, k, 2,1\right)
\end{aligned}
$$


La primera sumatoria representa el valor total de los componentes y servicios productivos incorporados al bien $\mathrm{m}^{*}$; el segundo término es la utilidad de la empresa ubicada en México, finalmente, la última sumatoria, mide el valor de los componentes y servicios productivos extranjeros registrados en el programa maquilador que se sustraen del valor agregado. Conviene enfatizar que cl último componente no excluye necesariamente todos los insumos y materiales de manufactura extranjera que intervienen directa o indirectamente en el proceso productivo. En las plantas localizadas en ciudades fronterizas, especialmente los de la zona libre, algunos de los gastos registrados como locales se efectúan realmente en el lado norte de la frontera y representan una importación que no debcría aparecer en el concepto cl valor agregado. Una medición correcta debcría cxcluir nosólo los bienes importados autorizados, sino todos los que se utilizan en la producción; la medición actual sobrecstima el valor agregado porque se basa en un criterio administrativo estrecho de lo que constituye una importaciòn.

\section{Estadísticas y definición de maquiladoras en Estados Unidos:}

El criterio seguido por Estados Unidos para reportar información sobre la coproducción internacional, se ciñe a las disposiciones de las fracciones 806.30 y 807.00 del Código de Impuestos al Comercio Exterior de Estados Unidos. Ambas fracciones se refieren a exportaciones temporales e insumos estadunidenses para la reimportación, después de haber pasado por un proceso de transformación o ensamble. Más en concreto, la fracción 806.30 se refiere a productos metálicos (excluyendo los preciosos) manufacturados en Estados Unidos, exportados para su procesamiento y reimportados para recibir un tratamiento final; la base gravable de estos artículos es solamente el valor del procesamiento externo. La fracción 807 es más general, ya que se refiere a toda clase de productos, no sólo a los metálicos, pero reduce el proceso de transformaciòn al ensamble de artículos que no pierden sus características físicas y no reciben un tratamiento que aumente su valor individual (exceptuando la limpieza, lubricación o pintura). Estos productos se gravan a su reingreso de acuerdo a su valor total menos el valor de los componentes manufacturados en Estados Unidos.

Como en el caso de los datos mexicanos, las fuentes estadounidenses registran instancias de produción internacional conjunta si 
existe un bien no terminado $i^{*}$, producido en Estados Unidos y reimportado en forma del bien $\mathrm{m}^{*} \mathrm{y}$ recibe un tratamiento que exenta del pago de impuestos de reimportación al valor de $\mathrm{i}^{*}$ (es decir 1=2). En forma de ecuación se puede escribir:

$$
\text { (7) } X\left(i^{*}, m^{*}, 3, k, n, 2\right) \cdot Q\left(m^{*} 3, k, 2\right)>0
$$

para algún valor de $\mathrm{k}$ y de $\mathrm{n}$.

Esta definición de conceptos tampoco garantiza que los datos se refieran a subcontratación en sentido estricto, menos aún que se refieran a actividades de maquila, aunque sí exige que la contraparte del contrato sea internacional.

El criterio de selección de datos requiere que las actividades se acoplen a los requisitos impuestos por el Código de Impuestos de Estados Unidos, que no fue diseñado para identificar los casos de exportación temporal para la exportación, sino para discriminar entre aquellos que ameritan un tratamiento de excepción y los que no. La legislación aduanera mexicana, en franco contraste con la estadounidense, sí busca amparar virtualmente todos los casos de transferencia internacional temporal para el procesamiento. La cobertura del fenómeno debe ser mayor en las estadísticas mexicanas, ya que las de Estados Unidos omiten conscientemente algunas de estas transacciones.

La fracción 806.30 recibe un tratamiento burocrático semejante al de los programas maquiladores de México, ya que antes de exportar es necesario llevar una forma de registro describiendo los artículos y el nombre del fabricante de Estados Unidos, indicando qué empresa completará el proceso al ser reimportadas las partes, con la aprobación de los oficiales de aduanas. En ambos casos, el proceso se avala con un registro administrativo que muestra que se cumplen los requisitos formales para recibir los beneficios del tratamiento arancelario especial. En contraste, el uso de la fracción 807 se consuma al reimportar las partes que salieron temporalmente de Estados Unidos; por lo tanto, no es necesario registrar el programa de coproducción a su inicio. Sin embargo, la principal diferencia entre las fracciones 806 y 807 reside en la forma de calcular la base gravable. La fracción 806 grava solamente el valor agregado externo, mientras que la 807 grava el valor total menos el valor de los componentes de Estados Unidos; en el primer caso no es necesario conocer el precio de los componentes 
ya procesados (que es la base para calcular el valor total), sino solamente los costos y utilidades generadas fuera del país; en el segundo caso ambos son necesarios. La estimación de los precios de bienes semiterminados presenta graves problemas que frecuentemente llevan a la subestimación. Un método ampliamente utilizado consiste en multiplicar la suma del valor agregado externo y de las partes estadounidenses por una tasa de mark up para asignar un valor a las utilidades, los servicios de asesoría, capital y la depreciación que normalmente no se contabilizan en el componente externo ni en las partes originales.

En los símbolos, el valor agregado de la empresa $\left(3, k^{*}\right)$, que es el valor gravable, para efectos de los productos metálicos se calcula de la siguiente manera:

$$
\begin{aligned}
& \text { (8) VA }\left(m^{*}, 3, k^{*}, l\right)=\sum_{i=1}^{I} \sum_{n=1}^{N} \sum_{l=1}^{L} X\left(i, m^{*}, 3, k^{*}, n, l\right) \\
& +K\left(i, m^{*}, 3, k^{*}, n, l\right)+A\left(i, m^{*}, 3, k^{*}, n . l\right) \\
& +T\left(i, m^{*}, 3, l\right)+S\left(i, m^{*}, 3, l\right)+F\left(i, m^{*}, 3, l\right) \\
& -\sum_{i=1}^{I} \sum_{n=1}^{N} \quad X\left(i, m^{*}, 3, k^{*}, n, l\right)+U\left(m^{*}, 3, k^{*}, l\right)
\end{aligned}
$$

Es decir, se calcula como la suma del valor monetario de todos los materiales y partes utilizadas en la transformación del bien $\mathrm{m}^{*}$, excluyendo los bienes $\mathrm{i}^{*}$ exentos del impuesto de reimportación $(I=1)$, los servicios de capital y asesoría tecnológica y administrativa ( en ocasiones estimado como un porcentaje fijo de los demás componentes del valor agregado), el trabajo, los servicios públicos, los fletes, seguros y trámites, y la utilidad obtenida por la empresa que realiza el procesamiento fuera de Estados Unidos. El segundo sumando de la ecuación (8) representa el valor de las partes no sujeta al pago de aranceles que se registra en las estadísticas como el componente nogravable. El componente de lautilidad más que un dato objetivo del beneficio económico de la actividad es generalmente un valor imputado en la aduana. Cuando no se conoce el valor de los servicios de capital, de las asesorías y de las utilidades para la empresa 
procesadora fuera de Estados Unidos, la ecuación(8) se aproxima con la siguiente fórmula :

$$
\begin{aligned}
& \text { (9) } \operatorname{VA}\left(\mathrm{m}^{*}, 3, \mathrm{k}^{*}, \mathrm{l}\right)=\sum_{\mathrm{i}=1}^{\mathrm{I}} \sum_{\mathrm{n}=1}^{\mathrm{N}} \sum_{\mathrm{l}=1}^{\mathrm{L}} \mathrm{X}\left(\mathrm{i}, \mathrm{m}^{*}, 3, \mathrm{k}^{*}, \mathrm{n}, \mathrm{l}\right) \\
& +\mathrm{T}\left(\mathrm{i}, \mathrm{m}^{*}, 3\right)+\mathrm{S}\left(\mathrm{i}, \mathrm{m}^{*}, 3\right)+\mathrm{F}\left(\mathrm{i}, \mathrm{m}^{*}, 3\right)-\sum_{\mathrm{i}=1}^{\mathrm{I}} \sum_{\mathrm{n}=1}^{\mathrm{N}} \\
& \left.\mathrm{X}\left(\mathrm{i}, \mathrm{m}^{*}, 3, \mathrm{k}^{*}, \mathrm{n}, \mathrm{l}\right)+(\mathrm{l}) \mathrm{r}\left(\mathrm{m}^{*}, 3\right)\right)
\end{aligned}
$$

donde la variable $\mathrm{r}\left(\mathrm{m}^{*}, 3\right)$ es una tasa (mark up) que imputa un valor a las variables de las que no se cuenta con una estimación directa. Este procedimiento administrativo tiene como objetivo reducir la tendencia a subvaluar los flujos de retorno en el proceso de producción internacional conjunto. La apreciación general es que no se ha logrado eliminar este problema totalmente.

En contraste, el valor gravable para la fracción 807 se calcula restando el valor de los componentes aceptados del valor total de los bienes reimportados. La fórmula de estimación es la siguiente:

$$
\begin{aligned}
& \text { (10) } \mathrm{VG}\left(\mathrm{m}^{*}, 3, \mathrm{k}^{*}, \mathrm{l}\right)=\mathrm{P}\left(\mathrm{m}^{*}, 3, \mathrm{k}^{*}, \mathrm{l}\right) \cdot \mathrm{q}\left(\mathrm{m}^{*}, 3, \mathrm{k}^{*}, \mathrm{l}\right) \\
& -\sum_{\mathrm{i}=1}^{\mathrm{I}} \sum_{\mathrm{n}=1}^{\mathrm{N}} \mathrm{X}\left(\mathrm{i}, \mathrm{m}^{*}, 3, \mathrm{k}^{*}, \mathrm{n}, \mathrm{l}\right)
\end{aligned}
$$

La ecuación (10) se basa en el valor de los productos al entrar a Estados Unidos, sustrayendo el valor de los componentes exentos de impuesto de reimportación. La dificultad que presenta esta ecuación es que requiere conocer el precio del bien en Estados Unidos, siendo que la mayoría de los bienes son intermedios yse negocian en mercados cerrados o son transacciones entre empresas filiales y matrices. En muchos bienes es posible asignar un precio más o menos adecuado; en otros, el valor total se aproxima con el valor agregado total incluyendo los componentes estadounidenses a los que se impone una tasa (mark up) para incluir la utilidad, no de la empresa fuera de Estados Unidos (que se incorpora al VA $\left(\mathrm{m}^{*}, 3, \mathrm{k}^{*}, 1\right)$ en la ecuación (8), sino de la compañía matriz estadounidense. Esta tasa multiplicativa 
$\mathrm{r}\left(\mathrm{m}^{*}, 3\right)$ también incluye la compensación por servicios de capital y la asesoría tecnológica y administrativa. Por lo tanto, la ecuación (10) se calcula como :

(11) $\mathrm{VG}\left(\mathrm{m}^{*}, 3, \mathrm{k}^{*}, \mathrm{l}\right)=\left[1+\mathrm{r}\left(\mathrm{m}^{*}, 3\right)\right] . \mathrm{VA}\left(\mathrm{m}^{*}, \mathrm{k}^{*}, 3\right)$

$$
+\sum_{i=1}^{I} \sum_{n=1}^{N} x\left(i, m^{*}, 3, k^{*}, n, l\right)
$$$$
-\sum_{i=1}^{I} \sum_{n=1}^{N} X\left(i, m^{*}, 3, k^{*}, n, 1\right)
$$

Para estimar el impuesto de importación de acuerdo a las tarifas 806.30 se multiplica el valor agregado $\mathrm{VA}\left(\mathrm{m}^{*}, 3, \mathrm{k}^{*}, 1\right)$ por la tarifa $t\left(m^{*}\right)$; para la tarifa 807 se multiplica el valor gravable $V G\left(m^{*}, 3, k^{*}, l\right)$ por la tasa $t\left(\mathrm{~m}^{*}\right)$. Es decir, cambian tanto el procedimiento de estimación de la tasa gravable como la naturaleza y, por lo tanto, la tasa de gravamen de los bienes que se amparan por cada una de las fracciones anteriores.

\section{Discrepancias entre la información mexicana y estadounidense:}

Las observaciones anteriores, sobre los conceptos de producción internacional conjunta y las formas alternativas de medición de indicadores y conceptos, cobran importancia cuando se observa que las estadísticas estadounidenses de coproducción en México reportan un valor agregado dos veces mayor al de las cifras mexicanas. Nuestra apreciación del fenómeno es muy distinta si adoptamos una u otra fuente. Esta diferencia es especialmente grave si tomamos en consideración que las cifras estadounidenses tienden a subestimar la magnitud del valor agregado, que constituye la base gravable, o una parte de ella según la fracción arancelaria.

La discrepancia en la estimación de indicadores aparentemente tan claros, se puede explicar cuando menos parcialmente, por diferencias en conceptos, convenciones de agregación, criterios de selección de información y rezagos en el registro de los datos. La diferencia en los conceptos de valor agregado surge del uso final que 
se da a la información en ambos países; en México es un indicador del valor del producto generado localmente, mientras que en Estados Unidos es una medida del valor gravable. En México se elimina del valor agregado todas las partes y materiales de manufactura externa incorporadas y aceptadas en el programa maquilador de las empresas; en Estados Unidos se eliminan aquellas partes producidas en ese país que estén cubiertas por las fracciones arancelarias 806/807. Los criterios que sigue cada uno de los dos países para excluir materiales son distintos y obedecen a nociones particulares de lo que constituye un programa internacional de producción conjunta. El hecho de que los programas maquiladores mexicanos sean muy flexibles en cuanto a la importación de materiales y accesorios (además de herramientas y bienes de capital), y que las fracciones $806 / 807$ se ciñan a partes y componentes muestra que las deducciones al valor agregado autorizados por México son mayores. Esto contribuye a explicar las diferencia en los datos.

El segundo factor explicativo de las diferencias en el valor agregado es que el marco de referencia es distinto para los dos grupos de estadísticas. En México se estima el valor generado en el país medido en términos de los costos de factores y partes nacionales, y las utilidades a la operación local. En contraste, la fracción 807 de Estados Unidos estima el valor gravable (que se usa como indicador del valor agregado) no en base a los costos y las utilidades generados en el exterior, sino en base al valor del bien en Estados Unidos; este criterio lleva a agregar costos ocultos como investigación y desarrollo, asesorías, depreciación, discño y capital, además de las utilidades de la empresa estadounidense. Estas consideraciones contribuyen a explicar las discrepancias estadísticas entre las dos fuentes y, si fueran laúnica causa de problema, permitirían hacer una ricainterpretación de las ganancias de operación a ambos lados de la frontera.

El tercer factor que explica las diferencias estadísticas es el de los criterios de selección. México incluye solamente las operaciones de aquellas empresas que tienen programas maquiladores aceptados, ignorando a las que importan temporalmente para exportar partes cubiertas en las fracciones $806 / 807$ sin estar registrados como maquiladoras. La magnitud de la subestimación por este concepto es extremadamente difícil de precisar. La base de recolección mexicana puede ser incompleta. Sin embargo, el problema opucsto se puede presentar aún con mayor frecuencia; es decir, cmpresas que aparecen en el registro de maquiladoras que no operan con productos avalados 
por las fracciones 806/807. Cuando esta situación se presenta, los datos de Estados Unidos subestiman la magnitud de la producción internacional conjunta, ya que ignoran todas las actividades que no se ajustan a las limitadas fracciones arancelarias.

El ultimo factor que se cita es que existe un rezago distinto en Méxicoy Estados Unidos para registrar la información de transacciones comunes entre los dos países. Esto difícilmente puede explicar una parte sustancial de las discrepancias estadísticas, ya que el sesgo originado por el rezago se da en la dirccción opuesta a la observada. En una actividad maquiladora dinámica en constante crecimiento, el país que tenga el menor rezago mostrará magnitudes menores en el valor agregado. Si, como cabría esperar, Estados Unidos procesa la información más rápidamente que México, el sesgo en las estadísticas favorecería a las estadísticas estadounidenses tal y como se observa. Sin embargo, este factor difícilmente puede considerarse como una explicacion completa de las discrepancias estadísticas observadas.

Los primeros tres factores explicativos se pueden deducir de un examen ciudadoso de las ecuaciones (6) y (8). Hay importantes diferencias en: el índice que se utiliza como control de agregación del valor total producido; en el índice que se usa para controlar el valor de los componentes importados, y las variables que se sustraen del valor para obtener el estimador del valor agregado.

\section{Producto nacional y Producto interno maquilador :}

La subcontratación internacional es una de las áreas en que es más importante la distinción entre producto internoy producto nacional. En las páginas anteriores se ha utilizado el concepto de valor agregado del Instituto Nacional de Estadística, Geografía e Informática que se define como la suma de salarios, intereses, rentas, alquileres, gastos en servicios públicos, gastos en trámites, transporte y mantenimiento más el valor de los insumos nacionales. Esta definición no se adecúa rigurosamente al concepto de valor agregado, ya que involucra tanto el pago a servicios productivos y el remanente de explotación como el valor de servicios y componentes; estos últimos representan la producción de otras empresas y sobreestiman el verdadero valor generado directamente en la planta maquiladora. La definición anterior no representa el valor agregado, sino una aproximación de los ingresos que deriva México de la subcontratación que, bajo ciertas condiciones, es igual a la suma de valores agregados que se integran 
en el producto exportado tomando en consideración la producción directa e indirecta y los servicios.

Conviene aclarar que esa definición no es absoluta, sino que se basa en el criterio de producto interno; es decir, la definición del valor agregado mide el producto (oienes y servicios) generado por factores nacionales y no nacionales dentro del territorio nacional mexicano, que redundan en la exportación de las llamadas maquiladoras. Su monto es diferente al que resultaría de medir el producto de las maquiladoras generado con factores nacionales dentro y fuera de México; es decir, el producto nacional maquilador. En las condiciones actuales, en que los factores nacionales tienen si acaso una escasa participación en la subcontratación fuera de México,mientras que los factores extranjeros participan extensamente en la subcontratación en el país, el producto interno es mayor a el nacional; por lo tanto, la medición del valor agregado sobreestima la contribución real de los factores nacionales al producto exportado y sobreestima la cantidad de divisas libres para el país. La diferencia entre las dos estimaciones indica las remesas susceptibles de salir del país por concepto de utilidades, intereses, alquileres, etc., obien, el monto en que aumentan los activos externos ubicados en México.

La estimación actual del valor agregado en las maquiladoras debe complementarse por medio del estudio de los acervos de capital e inversión extranjera y del análisis de la circulación de divisas en la industria. Una forma de corregir el valor agregado para reflejar el producto nacional consiste en modificar ligeramente el concepto de valor agregado exportado de la ecuación (6). Si consideramos únicamente los factores nacionales de un bien $\mathrm{m}^{*}$ que se exporta a $\mathrm{j}^{*}$ esa relación se transforma en:

$$
\begin{aligned}
& \text { (12) VAN }\left(\mathrm{m}^{*} \mathrm{j}^{*}, \mathrm{k}, 1\right)=\sum_{\mathrm{i}=1}^{\mathrm{I}} \sum_{\mathrm{j}=1}^{\mathrm{J}} \sum_{\mathrm{k}=1}^{\mathrm{K}} \sum_{\mathrm{n}=1}^{\mathrm{N}} \sum_{\mathrm{l}=1}^{\mathrm{L}} \mathrm{X}\left(\mathrm{i}, \mathrm{m}^{*} \mathrm{j}^{*}, \mathrm{k}, \mathrm{n}, \mathrm{l}\right)+ \\
& \mathrm{C}\left(\mathrm{i}, \mathrm{m}^{*} \mathrm{j}, \mathrm{k}, \mathrm{n}, \mathrm{l}\right)+\mathrm{A}\left(\mathrm{i}, \mathrm{m}^{*} \mathrm{j}, \mathrm{k}, \mathrm{n}, \mathrm{l}\right)+\mathrm{T}\left(\mathrm{i}, \mathrm{m}^{*} \mathrm{j}, \mathrm{l}\right) \\
& +\mathrm{S}\left(\mathrm{i}, \mathrm{m}^{*}, \mathrm{j}, \mathrm{l}\right)+\mathrm{F}\left(\mathrm{i}, \mathrm{m}^{*}, \mathrm{j}, \mathrm{l}\right)+\mathrm{U}\left(\mathrm{m}^{*}, \mathrm{j}, \mathrm{k}, \mathrm{l}\right)
\end{aligned}
$$




$$
\begin{aligned}
& \sum_{i=1}^{I} \sum_{j=3}^{4} \sum_{k=1}^{K} \sum_{n=1}^{N} \sum_{l=1}^{L} X\left(i, m^{*}, j, k, n, l\right)+C\left(i, m^{*}, j, k, n, l\right) \\
& A(i, m, j, k, n, l) \\
& -\sum_{i=1}^{I} \sum_{j=3}^{4} \sum_{k=1}^{K} \sum_{1-1}^{L} T\left(i, m^{*}, j, l\right)+S\left(i, m^{*}, j, l\right)+F\left(i, m^{*}, j, l\right)+U\left(m^{*}, j, k, l\right)
\end{aligned}
$$

Es decir, al valor total de las partes, componentes, servicios y utilidades se le resta no sólo el valor de los componentes, asesorías y scrvicios de capital importados bajo el régimen de maquiladora, sino también el valor del trabajo, servicios públicos, fletes, seguros y trámites imputables a la producción en México pero que provienen del exterior, y los componentes asesorías y servicios de capital que entran fuera del régimen de maquiladora.

El cociente entre VA $\left(\mathrm{m}^{*}\right)$ y VAN $\left(\mathrm{m}^{*}\right)$ representa la relación entre el producto interno y el producto nacional del sector maquilador. Mientras más difiera de uno este valor menos eficaz es, ceteris paribus, el ingreso de divisas que genera el sector. Cabe señalar que la variable $\operatorname{VAN}\left(\mathrm{m}^{*}\right)$ se puede aproximar utilizando datos sobre inversión extranjera directa en las maquiladoras y con datos más precisos sobre la composición de la fuerza de trabajo y el capital productivo de acuerdo a la nacionalidad de los factores.

En general es deseable calcular los conceptos de producto internacional $\mathrm{y}$ producto interno de todas las actividades productivas. Es posible ligar el bienestar y la fortaleza de la economía con aquella parte del producto que es, a la vez, internoy nacional; es decir, aquella parte de la producción hecha en México que se obtiene con factores propiedad de agentes nacionales. La parte del producto en que no se da la coincidencia de los dos criterios se presta a problemasgraves desde el punto de vista de la evaluación de su efecto en el bienestar de los nacionales; aquellas actividades en que el producto interno excede al nacional implica dependencia de recursos externos y una eventual, cuando no permanente, salida de recursos financieros y/o producto; aquellas actividades en que el producto nacional excede al interno implica una fuga presente o pasadade recursos que puede llevar o bien a un ingreso presente o futuro de recursos financieros y/o producto externo para el país, o bien, el riesgo de un eventual cambio de nacionalidad del recurso. 
El problema es, en gran medida, de costo de oportunidad ipodría existir la actividad sin la participación de recursos cxternos $\mathrm{cn}$ cl caso en que el producto interno excede al nacional? o iqué podrían producir localmente los recursos nacionales que han salido de los límites geográficos en el caso de las actividades en que el producto nacional excede al interno?

Además, existe la complicación creada por los efectos distributivos entre los factores productivos de la participación en las actividades de factores internos y en la salida de factores nacionales. 
ESTRUCTURA DE LA INDUSTRIA MAQUILADORA DE EXPORTACION

\section{B I B L I O G R A F I A}

CARRILLO, Jorge y Alberto Hernández, 1985. Mujeres fronterizas en la industria maquiladora, SEP-CEFNOMEX, México.

INEGI, 1986. Estadística de la industria maquiladora de exportación 1975-1985, México.

GRUNWALD, Joseph y Kenneth Flamm, 1985. The global factory: foreing assembly in intemational trade, The Brooking Institution, Washington.

MARTINEZ-TARRAGO, Trinidad, et al., La industria maquiladora de exportación en las zonas fronterizas del norte de México, CIDE, México, s.t. (mecanoescrito).

TAMAYO, Jesús y José Luis Fernández, 1983. "La industria maquiladora en México", en zonas fronterizas: México-Estados Unidos, CIDE, México, (Colección Estudios Políticos 2).

UNITED States International Trade Commission, 1986. The impact of increased United States-México trade on douthwest border development, USTTC Publication 1915 . Washington, Noviembre 1986.

\section{Documentos:}

"Fragmentos del informe de la Comisión de Aranceles de Estados Unidos sobre las Industrias Maquiladoras de Exportación", en Comercio exterior, vol. 21, No. 4, abril de 1971. 